\title{
On incompleteness of bond markets with infinite number of random factors *
}

\author{
Michał Barski \\ Faculty of Mathematics, Cardinal Stefan Wyszyński University in Warsaw, Poland \\ Faculty of Mathematics and Computer Science, University of Leipzig, Germany \\ Michal.Barski@math.uni-leipzig.de \\ Jacek Jakubowski \\ Institute of Mathematics, University of Warsaw, Warsaw, Poland \\ J.Jakubowski@mimuw.edu.pl and \\ Jerzy Zabczyk \\ Institute of Mathematics, Polish Academy of Sciences, Warsaw, Poland \\ zabczyk@impan.pl
}

\begin{abstract}
The completeness of a bond market model with infinite number of sources of randomness on a finite time interval in the Heath-Jarrow-Morton framework is studied. It is proved that the market is not complete. A construction of a bounded contingent claim, which can not be replicated, is provided.
\end{abstract}

Key words: bond market, completeness, infinite dimensional model

AMS Subject Classification: 91B28, 91B70, 91B24.

JEL Classification Numbers: G10,G11

*The authors express thanks to the anonymous reviewers for their valuable comments which were helpful while improving the early version of the paper.

Research supported by Polish KBN Grant P03A 03429 „Stochastic evolution equations driven by Lévy noise” 


\section{Introduction}

We investigate the completeness of a continuous time zero-coupon bond market model in the Heath-Jarrow-Morton framework, see Heath, Jarrow and Morton (1992). Let $P(t, T)$, $0 \leq t, T \leq \bar{S}<\infty$ be the price at time $t$ of a bond paying 1 at maturity $T$ and $\hat{P}(t, T)$ be the discounted price; $\bar{S}$ denotes a finite time horizon of the model. The paper is devoted to the problem of completeness of the market. We say that a market is complete if an arbitrary bounded random variable can be replicated by an admissible strategy. Thus we are concerned with a problem of replicating for, each $S \leq \bar{S}$, contingent claims depending on the information available up to time $S$. If $S=\bar{S}$ we have a situation which occurs in practice, when a trader hedges a payoff which depends on the bond's prices up to time $\bar{S}$, uses bonds with maturities not exceeding $\bar{S}$. Strategies of this type will be called natural.

The bond market we study is infinite in the sense that the price process is a function-valued process. The concept of the portfolio and the strategy may be formalized in many possible ways. We base our approach on the stochastic integration theory with respect to the Hilbert space-valued martingales, as presented in Métivier (1982) and Da Prato and Zabczyk (1992).

The problem of completeness has been investigated by several authors. Björk, Di Masi, Kabanov and Runggaldier (1997) regarded the price process in the space of continuous functions and obtained conditions for approximate completeness. Cylindrical integration theory, due to Mikulevicius and Rozovskii (1998), Mikulevicius and Rozovskii (1999), and signed measures as portfolios were used in De Donno and Pratelli (2004). The problem of replicating with the help of natural strategies was discussed and partially solved in Carmona and Tehranchi (2004). It was shown in Carmona and Tehranchi (2004), with the use of the Malliavin calculus, that each contingent claim of a special form can be replicated. The problem was also considered in Aihara and Bagchi (2005). The lack of completeness was shown in Taflin (2005) however, for a non-standard definition of the set of contingent claims, namely for $D_{0}:=\bigcap_{p>1} L^{p}(\Omega)$.

In the present paper the problem of completeness in the class of all bounded random variables is studied. We consider the case when strategies take values in the space $G^{*}$ - the dual of the Sobolev space $G=H^{1}[0, \bar{S}]$. We prove that under some natural conditions, the bond market model is not complete. The main contribution of this paper is the result on incompleteness for bounded contingent claims. Moreover, we provide a construction of a bounded random variable which can not be replicated. As a corollary we obtain incompleteness when the strategies take values in $L^{2}[0, \bar{S}]$, compare Aihara and Bagchi (2005). Our results seem to be in a contrast with Theorem 4.1 in Aihara and Bagchi (2005) which states that the bond market is complete (see our Remark 3.9). At the end, we comment on solvability of the equation (3.28), crucial for the problem of market completeness. It turns out that we can solve (3.28) in the class of all integrable processes satisfying some natural condition, but the solutions do not have a natural financial interpretation. 


\section{The model}

\subsection{Process of bond prices}

We consider a bond market with a finite time horizon $\bar{S}$ defined on a filtered probability space $\left(\Omega, \mathcal{F},\left(\mathcal{F}_{t}\right)_{t \in[0, \bar{S}]}, \mathbb{P}\right)$ satisfying usual conditions. The filtration is generated by a sequence of independent standard Wiener processes $W^{i}, i=1,2, \ldots$, i.e. $\mathcal{F}_{t}$ is generated by $W^{i}(s)$, where $i=1,2, \ldots, s \leq t$. We interpret $W_{t}=\left(W^{1}(t), W^{2}(t), \ldots\right)$ as a cylindrical Wiener process $W$ in $l^{2}$. The dynamics of the forward rate curve $f$ is given in the form

$$
d f(t, T)=\alpha(t, T) d t+\sum_{i=1}^{\infty} \sigma^{i}(t, T) d W^{i}(t), \quad t \in[0, \bar{S}], T \in[0, \bar{S}],
$$

where we put

$$
\alpha(t, T)=\sigma^{i}(t, T)=0 \quad \text { for } t \geq T \text { and } i=1,2, \ldots
$$

The formula (2.1) defines a family of processes parametrized by a continuous parameter $T \in$ $[0, \bar{S}]$. For each $T \in[0, \bar{S}]$ the formula describes the evolution of the forward rate on the interval $[0, \bar{S}]$. Denote by $\sigma_{t}$ a linear operator from $l^{2}$ into $H:=L^{2}[0, \bar{S}]$ given by the formula

$$
\left(\sigma_{t} u\right)(T):=\sum_{i=1}^{\infty} \sigma^{i}(t, T) u^{i}, \quad u=\left(u^{i}\right)_{i=1}^{\infty} \in l^{2}, \quad t, T \in[0, \bar{S}]
$$

and $\alpha_{t}(T)=\alpha(t, T)$. In this notation the formula (2.1) has the following form:

$$
d f_{t}=\alpha_{t} d t+\sigma_{t} d W_{t},
$$

or equivalently, using a stochastic integral:

$$
f_{t}=f_{0}+\int_{0}^{t} \alpha_{s} d s+\int_{0}^{t} \sigma_{s} d W_{s}, \quad t \in[0, \bar{S}] .
$$

For the concept of the stochastic integral in Hilbert spaces see Métivier (1982), Da Prato and Zabczyk (1992).

The drift coefficient $\alpha$ is assumed to be a process taking values in the space $H$, with the norm denoted by $|\cdot|_{H}$, satisfying Bochner integrability condition:

$$
\int_{0}^{\bar{S}}\left|\alpha_{t}\right|_{H} d t=\int_{0}^{\bar{S}}\left(\int_{0}^{\bar{S}} \alpha^{2}(t, T) d T\right)^{\frac{1}{2}} d t<\infty \quad \mathbb{P}-\text { a.s.. }
$$

Processes $\left(\sigma_{t}\right)$ is assumed to be a predictable process taking values in the space of HilbertSchmidt operators $L_{H S}\left(l^{2}, H\right)$ :

$$
\left\|\sigma_{t}\right\|_{L_{H S}\left(l^{2}, H\right)}^{2}=\sum_{i=1}^{\infty}\left|\sigma_{t}^{i}\right|_{H}^{2}=\sum_{i=1}^{\infty}\left(\int_{0}^{\bar{S}} \sigma^{i}(t, T)^{2} d T\right)<\infty \quad \mathbb{P}-\text { a.s. }
$$


and to satisfy the integrability condition:

$$
\int_{0}^{\bar{S}}\left\|\sigma_{t}\right\|_{L_{H S}\left(l^{2}, H\right)}^{2} d t=\sum_{i=1}^{\infty} \int_{0}^{\bar{S}}\left(\int_{0}^{\bar{S}} \sigma^{i}(t, T)^{2} d T\right) d t<\infty \quad \mathbb{P}-\text { a.s.. }
$$

Conditions (2.5) and (2.7) are necessary and sufficient for the forward rate curve process $\left(f_{t}\right)$ to be continuous in $H$.

The short rate process $r$ is defined by $r(t):=f(t, t)$ and the evolution of the money held in the savings account is given by the equation:

$$
d B(t)=r(t) B(t) d t
$$

The bond price $P$ is a process defined by the following formula:

$$
P(t, T)=e^{-\int_{t}^{T} f(t, u) d u},
$$

and the discounted bond price $\hat{P}$, due to $(2.2)$, satisfies:

$$
\hat{P}(t, T):=B^{-1}(t) P(t, T)=e^{-\int_{0}^{T} f(t, u) d u} .
$$

Let $G:=H^{1}[0, \bar{S}]$ be the Hilbert space of absolutely continuous functions with square integrable first derivative equipped with the norm:

$$
|g|_{G}^{2}:=|g(0)|^{2}+\int_{0}^{\bar{S}}\left(\frac{d g}{d s}(s)\right)^{2} d s, \quad g \in G .
$$

Note that the process $\hat{P}$ takes values in $G$. In fact, since:

$$
\frac{d}{d T} \hat{P}(t, T)=-\hat{P}(t, T) f(t, T)
$$

we have:

$$
\begin{aligned}
\left|\hat{P}_{t}\right|_{G}^{2} & =\hat{P}(t, 0)^{2}+\int_{0}^{\bar{S}}(\hat{P}(t, T) f(t, T))^{2} d T \\
& \leq \hat{P}(t, 0)^{2}+C(t) \int_{0}^{\bar{S}} f(t, T)^{2} d T<\infty
\end{aligned}
$$

where $C(t):=\sup _{T \in[0, \bar{S}]} \hat{P}^{2}(t, T)$ is finite because $\hat{P}(t, T)$ is a continuous function of $T$. We will also assume that the model is arbitrage-free, in the sense that the process $\hat{P}(\cdot, T)$ is a local martingale for every $T \in[0, \bar{S}]$, see Delbaen and Schachermayer (1994), Delbaen and Schachermayer (1998). This postulate is satisfied if and only if the following $H J M$-condition holds, see e.g. Heath, Jarrow and Morton (1992), Jakubowski and Zabczyk (2007):

$$
\int_{t}^{T} \alpha(t, u) d u=\frac{1}{2}\left|\int_{t}^{T} \sigma(t, u) d u\right|_{l^{2}}^{2} \forall t, T \in[0, \bar{S}] .
$$


Differentiating (2.10) with respect to $T$ gives the following formula:

$$
\alpha(t, T)=\sum_{i=1}^{\infty} \sigma^{i}(t, T) \int_{t}^{T} \sigma^{i}(t, u) d u \quad \forall t, T \in[0, \bar{S}] .
$$

As a consequence of (2.11) we can write the following expression for the process $\hat{P}$ :

$$
d \hat{P}(t, T)=\hat{P}(t, T)\left(\sum_{i=1}^{\infty} b^{i}(t, T) d W^{i}(t)\right),
$$

where

$$
b^{i}(t, T):=-\int_{0}^{T} \sigma^{i}(t, u) d u, \quad t, T \in[0, \bar{S}]
$$

Let $\left(\Gamma_{t}\right)$ be a stochastic process with values in the space of linear operators from $l^{2}$ into $G$ given by the formula:

$$
\left(\Gamma_{t} u\right)(T):=\hat{P}(t, T) \sum_{i=1}^{\infty} b^{i}(t, T) u^{i}, \quad u \in l^{2}, \quad t, T \in[0, \bar{S}] .
$$

Proposition 2.1 For every $t \in[0, \bar{S}], \Gamma_{t}$ given by (2.14) is a Hilbert-Schmidt operator form $l^{2}$ to G. Moreover, with probability one

$$
\int_{0}^{\bar{S}}\left\|\Gamma_{t}\right\|_{L_{H S}\left(l^{2}, G\right)}^{2} d t<\infty .
$$

Proof: At the beginning we show an auxiliary estimation. By (2.9) we have:

$$
|\hat{P}(t, T)| \leq e^{\int_{0}^{\bar{S}}|f(t, u)| d u} \leq e^{\left(\int_{0}^{\bar{S}}|f(t, u)|^{2} d u\right)^{\frac{1}{2}}(\bar{S})^{\frac{1}{2}}}=e^{\left|f_{t}\right|_{H}(\bar{S})^{\frac{1}{2}}} .
$$

Since $\left(f_{t}\right)$ is a continuous process in $H$, we conclude that the function $t \longrightarrow\left|f_{t}\right|_{H}$ is bounded as a continuous function on $[0, \bar{S}]$, i.e. there exists a constant $A>0$ such that:

$$
\sup _{t \in[0, \bar{S}]}\left|f_{t}\right|_{H} \leq A
$$

Thus for some $B>0$ we have:

$$
|\hat{P}(t, T)| \leq B \quad \forall t, T \in[0, \bar{S}] .
$$

For any $i=1,2, \ldots$, we have:

$$
\begin{aligned}
\frac{d}{d T}\left(\hat{P}(t, T) b^{i}(t, T)\right) & =\frac{d}{d T} \hat{P}(t, T) b^{i}(t, T)+\hat{P}(t, T) \frac{d}{d T} b^{i}(t, T) \\
& =f(t, T) \hat{P}(t, T) \int_{0}^{T} \sigma^{i}(t, u) d u-\hat{P}(t, T) \sigma^{i}(t, T) .
\end{aligned}
$$


Using (2.17), (2.18), 2.19) we can estimate the $G$-norm of $\hat{P}(t, \cdot) b^{i}(t, \cdot)$ by the $H$-norm of $\sigma_{t}^{i}$ :

$$
\begin{aligned}
& \left|\hat{P}(t, \cdot) b^{i}(t, \cdot)\right|_{G}^{2}=\int_{0}^{\bar{S}} \hat{P}(t, T)^{2}\left(f(t, T) \int_{0}^{T} \sigma^{i}(t, u) d u-\sigma^{i}(t, T)\right)^{2} d T \\
& \quad \leq B^{2} \int_{0}^{\bar{S}} 2\left(\left(f(t, T) \int_{0}^{T} \sigma^{i}(t, u) d u\right)^{2}+\sigma^{i}(t, T)^{2}\right) d T \\
& \quad \leq B^{2}\left(2 \int_{0}^{\bar{S}} f(t, T)^{2}\left(T \int_{0}^{T} \sigma^{i}(t, u)^{2} d u\right) d T+2\left|\sigma_{t}^{i}\right|_{H}^{2}\right) \\
& \quad \leq B^{2}\left(2 \int_{0}^{\bar{S}} f(t, T)^{2} \bar{S}\left|\sigma_{t}^{i}\right|_{H}^{2} d T+2\left|\sigma_{t}^{i}\right|_{H}^{2}\right) \\
& \quad \leq 2 B^{2}\left|\sigma_{t}^{i}\right|_{H}^{2}\left(\bar{S} \int_{0}^{\bar{S}} f(t, T)^{2} d T+1\right)=2 B^{2}\left|\sigma_{t}^{i}\right|_{H}^{2}\left(\bar{S}\left|f_{t}\right|_{H}^{2}+1\right) \\
& \quad \leq 2 B^{2}(\bar{S} A+1)\left|\sigma_{t}^{i}\right|_{H}^{2} .
\end{aligned}
$$

Let $\left(e^{i}\right)_{i=1}^{\infty}$ be a standard basis in $l^{2}$. In virtue of (2.7) and (2.20) we obtain the desired estimation:

$$
\begin{aligned}
& \int_{0}^{\bar{S}}\left\|\Gamma_{t}\right\|_{L_{H S}\left(l^{2}, G\right)}^{2} d t=\int_{0}^{\bar{S}} \sum_{i=1}^{\infty}\left|\Gamma_{t} e^{i}\right|_{G}^{2} d t=\int_{0}^{\bar{S}} \sum_{i=1}^{\infty}\left|\hat{P}(t, \cdot) b^{i}(t, \cdot)\right|_{G}^{2} d t \\
& \quad \leq 2 B^{2}(\bar{S} A+1) \int_{0}^{\bar{S}} \sum_{i=1}^{\infty}\left|\sigma_{t}^{i}\right|_{H}^{2} d t \leq 2 B^{2}(\bar{S} A+1) \int_{0}^{\bar{S}}\left\|\sigma_{t}\right\|_{L_{H S}\left(l^{2}, H\right)}^{2} d t<\infty .
\end{aligned}
$$

As an immediate consequence we obtain:

Corollary 2.2 The process $\hat{P}$ of discounted bond prices is a G-valued local martingale.

Since (2.15) holds, the equation (2.12) can be written in the form, see Métivier (1982), Da Prato and Zabczyk (1992):

$$
\hat{P}_{t}=\hat{P}_{0}+\int_{0}^{t} \Gamma_{s} d W_{s}, \quad t \in[0, \bar{S}]
$$

\subsection{Portfolios and strategies}

\subsubsection{Trading strategies}

In general portfolios $\varphi$ are identified with linear functionals acting on a space in which the price process lives. For the bond market the following classes of portfolios are considered in literature. 
A) Portfolios consisting of finite or infinite number of bonds:

$$
\varphi=\sum_{i=1}^{m} \alpha_{i} \delta_{\left\{T_{i}\right\}}, \quad m \in \mathbb{N} \cup \infty, \alpha_{i}, T_{i} \in[0, \bar{S}], i=1,2, \ldots, m, \sum_{i=1}^{\infty}\left|\alpha_{i}\right|<\infty .
$$

B) $\varphi$ are finite signed measures on the interval $[0, \bar{S}]$.

C) $\varphi$ are bounded functionals on the space $G=H^{1}[0, \bar{S}]$, shortly $\varphi \in G^{*}$, where $G^{*}$ denotes the dual space.

The class $(A)$ has an obvious interpretation. Some justification for using portfolios as finite signed measures or as elements of $G^{*}$ can be found in De Donno and Pratelli (2004) or in Björk, Di Masi, Kabanov and Runggaldier (1997) and Björk, Kabanov and Runggaldier (1997). Let us recall that the space $G^{*}$ contains finite signed measures on the interval $[0, \bar{S}]$.

Definition 2.3 A trading strategy is any predictable process with values in some fixed class in $(A)-(C)$.

Definition 2.4 The (discounted) wealth process $\hat{V}^{\varphi}$ corresponding to $\varphi$ is given by:

$$
\hat{V}_{t}^{\varphi}=\hat{V}_{0}^{\varphi}+\int_{0}^{t}<\varphi_{s}, d \hat{P}_{s}>_{G^{*}, G}, \quad t \in[0, \bar{S}]
$$

The concept of stochastic integral will be discussed now.

Since $\hat{P}$ is a $G$-valued local martingale of the form (2.21), the class of integrands, see Métivier (1982), consists of all $G^{*}$-valued predictable processes $\varphi$ satisfying:

$$
\int_{0}^{\bar{S}}\left|\varphi_{t}\left(Q_{t}^{\frac{1}{2}}\right)\right|_{G^{*}}^{2} d t<\infty
$$

where

$$
Q_{t}=\Gamma_{t} \Gamma_{t}^{\prime}, \quad t \in[0, \bar{S}]
$$

In (2.23) $\Gamma_{t}^{\prime}$ is the conjugate of $\Gamma_{t}$, i.e. for all $a \in G$ and $b \in l^{2}$ we have $<\Gamma_{t}^{\prime} a, b>_{l^{2}}=<a, \Gamma_{t} b>_{G}$. We say that a predictable process $\varphi$ is $\hat{P}$ integrable if $\varphi$ satisfies (2.22). Note that $\varphi$ can be $\hat{P}$ integrable although $P\left(P_{t} \in \operatorname{Dom} \varphi_{t}\right)=0$, so $<\varphi_{t}, P_{t}>$ is not defined. Thus from financial point of view it is natural to assume that $\varphi$ takes values in $G^{*}$ since $P$ lives in $G$. The construction of the stochastic integral in Métivier (1982) is developed for a square integrable martingale, but it can be extended to local martingales by the localization procedure. Moreover, identifying Hilbert space $G$ with its dual, with $\tilde{\varphi} \in G^{*}$ corresponding to $\varphi \in G$, we have

$$
\left|\varphi\left(Q_{t}^{\frac{1}{2}}\right)\right|_{G^{*}}^{2}=<\left(\Gamma_{t} \Gamma_{t}^{\prime}\right)^{\frac{1}{2}} \tilde{\varphi},\left(\Gamma_{t} \Gamma_{t}^{\prime}\right)^{\frac{1}{2}} \tilde{\varphi}>_{G}=\left|\Gamma_{t}^{\prime} \tilde{\varphi}\right|_{G}^{2}=\left|\Gamma_{t}^{*} \tilde{\varphi}\right|_{G^{*}}^{2}
$$


So, the condition (2.22) can be reformulated as:

$$
\int_{0}^{\bar{S}}\left|\Gamma_{s}^{*} \varphi_{s}\right|_{l^{2}}^{2} d s<\infty .
$$

Therefore, $\varphi$ is $\left(\hat{P}_{t}\right)$ integrable if (2.24) holds and then

$$
\int_{0}^{t}<\varphi_{s}, d \hat{P}_{s}>_{G^{*}, G}=\int_{0}^{t}<\Gamma_{s}^{*} \varphi_{s}, d W_{s}>_{l^{2}, l^{2}} .
$$

We define a class of admissible strategies in a standard way, see, for instance, Karatzas and Shreve (1998), Hunt and Kennedy (2005) and Jarrow and Madan (1991).

Definition 2.5 A trading strategy $\varphi$ is admissible if it is $\left(\hat{P}_{t}\right)$ integrable and if the process

$$
\int_{0}^{t}<\varphi_{s}, d \hat{P}_{s}>_{G^{*}, G}, \quad t \in[0, \bar{S}]
$$

is a martingale. The class of all admissible strategies will be denoted by $\mathcal{A}$.

Example 2.6 Assume that $\varphi$ is a $G^{*}$-valued predictable process.

1) If $\varphi$ satisfies the integrability condition:

$$
\mathbf{E}\left(\int_{0}^{\bar{S}}\left|\Gamma_{s}^{*} \varphi_{s}\right|_{l^{2}}^{2} d s\right)^{\frac{1}{2}}<\infty
$$

then $\varphi \in \mathcal{A}$. Indeed, the Burkholder-Davies-Gundy inequality implies that the integral $\int_{0}^{\cdot}<\varphi_{s}, d \hat{P}_{s}>_{G^{*}, G}$ is a martingale.

2) If there exists a constant $K>0$ such that the following condition holds:

$$
\left|\int_{0}^{t}<\varphi_{s}, d \hat{P}_{s}>_{G^{*}, G}\right|<K, \quad \forall t \in[0, \bar{S}]
$$

then $\varphi \in \mathcal{A}$, because a bounded local martingale is a martingale.

\section{Incompleteness}

\subsection{Incompleteness in general case}

We define the completeness of the market in a usual way.

Definition 3.1 Let $S \leq \bar{S}$. The bond market is complete on $[0, S]$ if for any $\mathcal{F}_{S}$-measurable, bounded random variable $\xi$ there exists an admissible strategy $\varphi$ and a constant $c$ such that

$$
\xi=c+\int_{0}^{S}<\varphi_{t}, d \hat{P}_{t}>_{G^{*}, G}
$$


Now we state the main theorem of the paper.

Theorem 3.2 The bond market is not complete on $[0, S]$ for any $S \leq \bar{S}$.

For the proof we will need the following lemmas.

Lemma 3.3 (Appendix B in Da Prato and Zabczyk (1992)) Let $X, Y, Z$ be three Hilbert spaces and $A: X \longrightarrow Z, B: Y \longrightarrow Z$ two linear bounded operators. Then $\operatorname{Im} A \subseteq \operatorname{Im} B$ if and only if there exists constant $c>0$ such that $\left\|A^{*} f\right\| \leq c\left\|B^{*} f\right\|$ for all $f \in Z^{*}$.

Lemma 3.4 Let $\xi$ be a square integrable, $\mathcal{F}_{S}$ measurable random variable such that $\mathbf{E} \xi=0$. Then $\xi$ can be represented in the following form:

$$
\xi=\int_{0}^{S}<\psi_{s}, d W_{s}>_{l^{2}, l^{2}}
$$

where $\psi$ is a predictable, $l^{2}$-valued process satisfying condition $\mathbf{E} \int_{0}^{S}\left|\psi_{s}\right|_{l^{2}}^{2} d s<\infty$. Moreover, $\mathbf{E} \xi^{2}=\mathbf{E} \int_{0}^{S}\left|\psi_{s}\right|_{l^{2}}^{2} d s$.

Proof: This result is true when $W$ is finite dimensional, see Lemma 18.11 in Kallenberg (2001). The generalization can be obtained by the following arguments. Let $\mathcal{G}_{n} \subseteq \mathcal{F}$ be a $\sigma$-field generated by

$$
W_{n}(s)=\left(W^{1}(s), W^{2}(s), \ldots, W^{n}(s)\right), \quad s \leq S,
$$

and let $\xi_{n}:=\mathbf{E}\left(\xi \mid \mathcal{G}_{n}\right)$. Since $\xi \in L^{2}(\Omega)$ and $\mathcal{G}_{n} \uparrow \mathcal{F}=\bigcup_{n \geq 1} \mathcal{G}_{n}$, so by the classical convergence theorem for martingales, see Corollary 7.22 and Theorem.7.23 in Kallenberg (2001), $\xi_{n} \longrightarrow \xi$ in $L^{2}(\Omega)$. On the other hand we have:

$$
\xi_{n}=\int_{0}^{S} \varphi_{n}(s) d W_{n}(s)
$$

Setting $\tilde{\varphi}_{s}^{n}:=\left(\varphi_{n}(s), 0, \ldots\right) \in l^{2}$ we see that

$$
\xi_{n}=\int_{0}^{S}<\tilde{\varphi}_{s}^{n}, d W_{s}>_{l^{2}, l^{2}}
$$

Hence $\mathbf{E}\left(\xi_{m}-\xi_{n}\right)^{2}=\mathbf{E} \int_{0}^{S}\left(\tilde{\varphi}_{s}^{n}-\tilde{\varphi}_{s}^{m}\right)^{2} d s \longrightarrow 0$ with $m, n \longrightarrow \infty$ and therefore $\left\{\tilde{\varphi}^{n}\right\}$ is a Cauchy sequence in $F=L^{2}\left(\Omega \times[0, S], \mathcal{F}_{S} \otimes \mathcal{B}[0, S], \mathbb{P} \times \lambda ; l^{2}\right)$. By the completeness of $F$ there exists a predictable limit $\varphi \in F$ of the sequence $\left(\tilde{\varphi}_{n}\right)$. Due to the fact that

$$
\int_{0}^{S}<\tilde{\varphi}_{s}^{n}, d W_{s}>_{l^{2}, l^{2}} \longrightarrow \int_{0}^{S}<\varphi_{s}, d W_{s}>_{l^{2}, l^{2}} \text { in } L^{2}(\Omega),
$$

we have the required representation: $\xi=\int_{0}^{S}<\varphi_{s}, d W_{s}>_{l^{2}, l^{2}}$.

The next lemma states a uniqueness of random variables representation in a class of admissible strategies. This property is crucial for the method which is used in the proof of the main result. 
Lemma 3.5 Let $x, y \in \mathbb{R}$. Assume that $\psi$ is integrable with respect to $W$ and such that $\int_{0}^{t} \psi_{s} d W_{s}, t \in[0, S]$ is a martingale. If for $\varphi \in \mathcal{A}$ the following condition

$$
x+\int_{0}^{S}<\varphi_{s}, d \hat{P}_{s}>_{G^{*}, G}=y+\int_{0}^{S}<\psi_{s}, d W_{s}>_{l^{2}, l^{2}},
$$

is satisfied, then $x=y$ and $\Gamma_{s}^{*} \varphi_{s}=\psi_{s}$ a.s. wrt. $P \otimes \lambda$ on $\Omega \times[0, S]$, where $\lambda$ denotes $a$ Lebesgue measure.

Proof: Taking expectations of both sides in (3.27) we immediately obtain that $x=y$.

Thus the following condition is satisfied:

$$
\int_{0}^{S}<\Gamma_{s}^{*} \varphi_{s}-\psi_{s}, d W_{s}>_{l^{2}, l^{2}}=0 .
$$

By Lemma 10.15 in Da Prato and Zabczyk (1992), there exists a standard, one dimensional Wiener process $B$ for which we have:

$$
\int_{0}^{t}<\Gamma_{s}^{*} \varphi_{s}-\psi_{s}, d W_{s}>_{l^{2}, l^{2}}=\int_{0}^{t}\left|\Gamma_{s}^{*} \varphi_{s}-\psi_{s}\right|_{l^{2}} d B(s) \quad \forall t \in[0, S] .
$$

Thus the process $\int_{0}^{t}\left|\Gamma_{s}^{*} \varphi_{s}-\psi_{s}\right|_{l^{2}} d B(s)$ is a martingale which is equal to zero at time $S$. So, it is equal to zero for every $t \in[0, S]$. By the uniqueness of the martingale representation, see Theorem 18.10 in Kallenberg (2001), we conclude that the integrand must be zero which implies: $\Gamma_{s}^{*} \varphi_{s}=\psi_{s}, P \otimes \lambda$ a.s.

In order to find a strategy which replicates a square integrable contingent claim $X$ one can use Lemma 3.4 to represent $X$ as

$$
X=E X+\int_{0}^{S}<\psi_{s}, d W_{s}>_{l^{2}, l^{2}},
$$

and then find an admissible strategy $\varphi$ by solving the following structural equation:

$$
\int_{0}^{S}<\varphi_{s}, d \hat{P}_{s}>_{G^{*}, G}=\int_{0}^{S}<\psi_{s}, d W_{s}>_{l^{2}, l^{2}}
$$

\subsubsection{Proof of Theorem 3.2}

Due to the definition of the integral with respect to $\hat{P}$, the set of all final portfolio values starting from zero initial endowment has the following structure:

$$
\left\{\int_{0}^{S}<\varphi_{t}, d \hat{P}_{t}>_{G^{*}, G}: \varphi \in \mathcal{A}\right\}=\left\{\int_{0}^{S}<\Gamma_{t}^{*} \varphi_{t}, d W_{t}>_{l^{2}, l^{2}}: \varphi \in \mathcal{A}\right\} .
$$

We will show that the operators $\Gamma_{t}^{*}, t \in[0, S]$, are not surjective and we construct process $\psi$ taking values in the space $l^{2}$ satisfying conditions: 
1) $\psi$ is integrable wrt. $W$ and such that $\int_{0}^{t}<\psi_{t}, d W_{t}>_{l^{2}, l^{2}}, t \in[0, S]$, is a martingale,

2) $\psi_{t} \notin \operatorname{Im}\left(\Gamma_{t}^{*}\right)$ with positive $\mathbb{P} \otimes \lambda$ measure,

3) the random variable $\int_{0}^{S}<\psi_{t}, d W_{t}>_{l^{2}, l^{2}}$ is bounded.

Then, in virtue of Lemma 3.5, for any $c \in \mathbb{R}$ and $\varphi \in \mathcal{A}$ we have:

$$
\int_{0}^{S}<\psi_{t}, d W_{t}>_{l^{2}, l^{2}} \neq c+\int_{0}^{S}<\Gamma_{t}^{*} \varphi_{t}, d W_{t}>_{l^{2}, l^{2}} .
$$

Thus the integral $\int_{0}^{S}<\psi_{t}, d W_{t}>_{l^{2}, l^{2}}$ is a bounded random variable which can not be replicated.

By Proposition 2.1 the operator $\Gamma_{t}$ is a Hilbert-Schmidt operator for any $t \in[0, S]$. Thus $\Gamma_{t}$ is compact, so is $\Gamma_{t}^{*}$. As a compact operator with values in infinite dimensional Hilbert space, $\Gamma_{t}^{*}$ is not surjective.

Let us consider the self-adjoint operator $\left(\Gamma_{t}^{*} \Gamma_{t}\right)^{\frac{1}{2}}: l^{2} \longrightarrow l^{2}$ which is also compact. For any $u \in l^{2}$ we have:

$$
\left|\left(\Gamma_{t}^{*} \Gamma_{t}\right)^{\frac{1}{2}} u\right|_{l^{2}}^{2}=<\left(\Gamma_{t}^{*} \Gamma_{t}\right)^{\frac{1}{2}} u,\left(\Gamma_{t}^{*} \Gamma_{t}\right)^{\frac{1}{2}} u>_{l^{2}}=<\Gamma_{t}^{*} \Gamma_{t} u, u>_{l^{2}}=<\Gamma_{t} u, \Gamma_{t} u>_{G}=\left|\Gamma_{t} u\right|_{G}^{2},
$$

so by Lemma 3.3 it follows that $\operatorname{Im}\left(\Gamma_{t}^{*}\right)=\operatorname{Im}\left(\left(\Gamma_{t}^{*} \Gamma_{t}\right)^{\frac{1}{2}}\right)$.

By Proposition 1.8 in Da Prato and Zabczyk (1992) the operator $\left(\Gamma_{t}^{*} \Gamma_{t}\right)^{\frac{1}{2}}$ can be represented by the formula:

$$
\left(\Gamma_{t}^{*} \Gamma_{t}\right)^{\frac{1}{2}}=\sum_{i=1}^{\infty} \lambda_{i}(t) g_{t}^{i} \otimes g_{t}^{i}
$$

where $\lambda_{i}(t)$ is a random variable and $g_{t}^{i}$ is an $l^{2}$ - valued random variable for $\mathrm{i}=1,2, \ldots$. Here " $\otimes$ " denotes the linear operation: $(a \otimes b) h=a<b, h>$ for $a, b, h \in l^{2}$. Moreover, $\lambda_{i}$ and $g^{i}$ are predictable as functions of $(\omega, t)$ and $\lambda_{i}(t) \longrightarrow_{i \rightarrow \infty} 0$ by compactness of $\left(\Gamma_{t}^{*} \Gamma_{t}\right)^{\frac{1}{2}}$.

Our aim now is to construct the process $\tilde{\psi}$ where $\tilde{\psi}_{t}=\left(\tilde{\psi}^{1}(t), \tilde{\psi}^{2}(t), \ldots\right) \in l^{2}$ such that it is not of the form $\sum_{i=1}^{\infty} \lambda_{i}(t) g_{t}^{i}<g_{t}^{i}, u>_{l^{2}}$ for any $u \in l^{2}$. This process must thus satisfy:

$$
\begin{aligned}
& \sum_{i=1}^{\infty}\left(\frac{\tilde{\psi}^{i}(t)}{\lambda_{i}(t)}\right)^{2}=\infty \\
& \sum_{i=1}^{\infty}\left(\tilde{\psi}^{i}(t)\right)^{2}<\infty
\end{aligned}
$$

Let us define the sequence $\left(i_{k}(t)\right)_{k=1,2, \ldots}$ in the following way:

$$
\begin{array}{r}
i_{1}(t):=\inf \left\{i: \frac{1}{\lambda_{i}(t)} \geq 1\right\}, \\
i_{k+1}(t):=\inf \left\{i>i_{k}: \frac{1}{\lambda_{i}(t)} \geq k\right\}
\end{array}
$$


and put

$$
\tilde{\psi}^{i}(t)= \begin{cases}0 & \text { if } i \neq i_{k}(t) \\ \frac{1}{k} & \text { if } i=i_{k}(t) .\end{cases}
$$

Then we have $\sum_{i=1}^{\infty}\left(\frac{\tilde{\psi}^{i}(t)}{\lambda_{i}(t)}\right)^{2} \geq \sum_{k=1}^{\infty} \frac{1}{k^{2}} k^{2}=\infty$ and $\sum_{i=1}^{\infty} \tilde{\psi}^{i}(t)^{2}=\sum_{k=1}^{\infty} \frac{1}{k^{2}}<\infty$, so the process is bounded in $l^{2}$. It is also predictable since it is obtained by measurable operations on predictable elements. Thus the process $\tilde{\psi}$ is integrable with respect to $W$ and $\int_{0}^{\cdot}<\tilde{\psi}, d W>_{l^{2}, l^{2}}$ is a martingale.

Now let us define a stopping time $\tau$ as:

$$
\tau:=\inf \left\{t>0:\left|\int_{0}^{t}<\tilde{\psi}_{t}, d W_{t}>_{l^{2}, l^{2}}\right| \geq 1\right\} \wedge S
$$

Finally, we define the required process as:

$$
\psi_{t}:=\tilde{\psi}_{t} \mathbf{1}_{[0, \tau)}(t)
$$

Remark 3.6 From Theorem 3.2 with $S=\bar{S}$ it follows that the bond market is not complete on $[0, \bar{S}]$ if traders can use natural strategies only.

\subsection{Comments on incompleteness in $L^{2}[0, \bar{S}]$}

In the paper Aihara and Bagchi (2005) strategies $\varphi$ with values in $L^{2}[0, \bar{S}]$, satisfying

$$
\mathbf{E}\left(\int_{0}^{\bar{S}}\left|\varphi_{t}\right|_{L^{2}[0, \bar{S}]}^{2} d t\right)<\infty
$$

are considered (see formula (4.2) in Aihara and Bagchi (2005)), and absolutely continuous measures are identified with their densities. With this definition of strategies it is shown that the market is complete (see Theorem 4.1. in Aihara and Bagchi (2005)). We have, however, the following proposition.

Proposition 3.7 There exists a model of the bond market in which all $L^{2}[0, \bar{S}]$-valued processes $\varphi$ satisfying (3.29) are admissible strategies.

Proof: We will construct the model by defining the volatility coefficient. Let us assume that $\tilde{\sigma}$ satisfies the following conditions:

$$
\begin{aligned}
& 0 \leq \tilde{\sigma}^{i}(t, T) \leq K \quad i=1,2, \ldots,(t, T) \in[0, \bar{S}] \times[0, \bar{S}] \text { for some } K>0 \\
& \left|\tilde{\sigma}_{t}^{i}\right|_{H}^{2} \leq \frac{1}{i^{2}} \quad i=1,2, \ldots, t \in[0, \bar{S}]
\end{aligned}
$$


and define a new operator $\sigma$ as

$$
\sigma^{i}(t, T)=\left\{\begin{array}{lll}
\tilde{\sigma}^{i}(t, T) & \text { if } \quad & \sum_{i=1}^{\infty} \int_{0}^{t} \tilde{\sigma}^{i}(s, T) d W^{i}(s) \geq 0 \\
0 & \text { if } \quad & \sum_{i=1}^{\infty} \int_{0}^{t} \tilde{\sigma}^{i}(s, T) d W^{i}(s)<0
\end{array}\right.
$$

Let the coefficient $\alpha$ be given by the HJM condition (2.11):

$$
\alpha(t, T)=\sum_{i=1}^{\infty} \sigma^{i}(t, T) \int_{t}^{T} \sigma^{i}(t, s) d s .
$$

It follows from (3.30), (3.31), (3.32) and (3.33) that coefficients $\alpha$ and $\sigma$ satisfy (2.5) and (2.7). Assume that the initial forward rate curve is nonnegative: $f(0, T) \geq 0$ for $T \in[0, \bar{S}]$. Then

$$
f(t, T)=f(0, T)+\int_{0}^{t} \alpha(s, T) d s+\sum_{i=1}^{\infty} \int_{0}^{t} \sigma^{i}(s, T) d W^{i}(s) \geq 0, \quad(t, T) \in[0, \bar{S}] \times[0, \bar{S}]
$$

and thus $\hat{P}(t, T)=e^{-\int_{0}^{T} f(t, u) d u} \leq 1$. It follows from the condition (3.31) that:

$$
\begin{aligned}
\sum_{i=1}^{\infty} \int_{0}^{\bar{S}} b^{i}(t, T)^{2} d T & =\sum_{i=1}^{\infty} \int_{0}^{\bar{S}}\left(\int_{0}^{T} \sigma^{i}(t, u) d u\right)^{2} d T \\
& \leq \sum_{i=1}^{\infty} \int_{0}^{\bar{S}}\left(T \int_{0}^{T} \sigma^{i}(t, u)^{2} d u\right) d T \leq \bar{S}^{2} \sum_{i=1}^{\infty} \frac{1}{i^{2}} \quad \forall t \in[0, \bar{S}] .
\end{aligned}
$$

As a consequence we obtain the following inequalities:

$$
\begin{aligned}
\mathbf{E}\left(\int_{0}^{\bar{S}}\left|\Gamma_{t}^{*} \varphi_{t}\right|_{l^{2}}^{2} d t\right) & \left.=\mathbf{E}\left(\int_{0}^{\bar{S}} \sum_{i=1}^{\infty}\left(\int_{0}^{\bar{S}} \varphi_{t}(T) \hat{P}(t, T) b^{i}(t, T) d T\right)^{2} d t\right)\right) \\
& \leq \mathbf{E}\left(\int_{0}^{\bar{S}}\left(\int_{0}^{\bar{S}} \varphi_{t}(T)^{2} d T \sum_{i=1}^{\infty} \int_{0}^{\bar{S}} b^{i}(t, T)^{2} d T\right) d t\right) \\
& \leq \bar{S}^{2} \sum_{i=1}^{\infty} \frac{1}{i^{2}} \mathbf{E}\left(\int_{0}^{\bar{S}} \int_{0}^{\bar{S}} \varphi_{t}(T)^{2} d T d t\right)<\infty .
\end{aligned}
$$

In view of (3.34) we conclude that for each $\varphi$ satisfying (3.29) the process $\int_{0}^{*}<\varphi_{t}, d \hat{P}_{t}>_{G^{*}, G}$ is a martingale and thus $\varphi$ is admissible.

Therefore it follows from Proposition 3.7 and Theorem 3.2 that

Corollary 3.8 There exists an incomplete bond market for which all $L^{2}[0, \bar{S}]$-valued processes satisfying (3.29) are admissible. 
Remark 3.9 Due to relation $L^{2}[0, \bar{S}]=H \supseteq G=H^{1}[0, \bar{S}]$ we can treat the process $\left(\hat{P}_{t}\right)$ as taking values in the space $H$. The inclusion $H^{*} \subseteq G^{*}$ expresses the fact, that if we admit $H$ as a state space for the discounted bonds prices, then the investor can use smaller class of strategies and as a consequence, in this case, the market is also incomplete. It follows from Corollary 3.8 that Theorem 4.1 in Aihara and Bagchi (2005) is false. It was pointed out by one of the reviewers that the market considered in Aihara and Bagchi (2005) is approximately complete but the limit passage, performed in the proof of Th. 4.1 to get completeness, is not correct.

\subsection{Comments on admissibility}

Notice that Lemma 3.5 can be reformulated in the following way. If for the $W$ integrable processes $\gamma, \psi$, such that the integrals $\int<\gamma_{s}, d W_{s}>_{l^{2}, l^{2}}$ and $\int<\psi_{s}, d W_{s}>_{l^{2}, l^{2}}$ are martingales, we have:

$$
x+\int_{0}^{S}<\gamma_{s}, d W_{s}>_{l^{2}, l^{2}}=y+\int_{0}^{S}<\psi_{s}, d W_{s}>_{l^{2}, l^{2}}
$$

for some $x, y \in \mathbb{R}$, then $x=y$ and $\gamma=\psi$. It turns out that this assertion is not true if we assume only the existence of the integrals or if we additionally assume that the integrals are bounded from below. A counterexample in a one dimensional case which we show is based on Example 8, page 237 in Lipcer and Shiryaev (2001).

Example 3.10 Let us consider a one dimensional Wiener process $B$ on the interval $[0,1]$. The following stopping time:

$$
\tau:=\inf \left\{t \in[0,1]: B^{2}(t)+t=1\right\}
$$

satisfies $P(0<\tau<1)=1$. The process

$$
X(t):=-\frac{2 B(t)}{\left(1-t^{2}\right)} \mathbf{1}_{\{t \leq \tau\}}
$$

is integrable with respect to $B$ because the following estimation holds:

$$
\int_{0}^{1} X(s)^{2} d s=4 \int_{0}^{\tau} \frac{B(s)^{2}}{(1-s)^{4}} d s<\infty .
$$

Applying the Itô formula to the process $\frac{B(t)^{2}}{(1-t)^{2}}$ we obtain:

$$
\int_{0}^{1} X(s) d B(s)-\frac{1}{2} X(s)^{2} d s=-1-2 \int_{0}^{\tau} B(s)^{2}\left(\frac{1}{(1-s)^{4}}-\frac{1}{(1-s)^{3}}\right) d s<-1 .
$$

As a consequence, the Doléans-Dade exponent $M=\mathcal{E}(X)$, which is a local martingale, is not a martingale because

$$
\mathbf{E}(M(1))=\mathbf{E}\left(e^{\int_{0}^{1} X(s) d B(s)-\frac{1}{2} X(s)^{2} d s}\right)<e^{-1}<M(0)=1 .
$$


The random variable $M(1)$ satisfies estimation $0<M(1)<e^{-1}$ and thus the application of the martingale representation theorem to the square integrable martingale $\mathbf{E}\left[M(1) \mid \mathcal{F}_{t}\right]$ provides:

$$
M(1)=\mathbf{E}[M(1)]+\int_{0}^{1} \gamma(s) d B(s)
$$

where $\mathbf{E} \int_{0}^{1} \gamma^{2}(s) d s<\infty$. On the other hand the application of the martingale representation theorem to the local martingale $M$ provides, see Theorem 18.10 in Kallenberg (2001):

$$
M(1)=M(0)+\int_{0}^{1} \psi(s) d B(s)=1+\int_{0}^{1} \psi(s) d B(s),
$$

where $P\left(\int_{0}^{1} \psi^{2}(s) d s<\infty\right)=1$. Moreover, $\psi$ satisfies condition $\mathbf{E} \int_{0}^{1} \psi^{2}(s) d s=\infty$ because $M$ is not a martingale.

Summarizing, we have two different representations of the same bounded random variable $M(1)$, i.e.

$$
\mathbf{E}[M(1)]=x \neq y=1 ; \quad \gamma \neq \psi
$$

Moreover, both representations are bounded from below by zero.

\section{Solvability of the structural equation}

As we can see the problem of market completeness is strictly connected with the existence of a solution to the structural equation (3.28). Theorem 3.2 shows that (3.28) may not have a solution in the class of $G^{*}$ - valued admissible strategies. However, the equation (3.28) can be considered in the class of all processes stochastically integrable with respect to $\hat{P}$. In the definition of the stochastic integral we follow Métivier (1982), see also Peszat and Zabczyk (2007). The class of integrands consists of all predictable processes $\varphi$ taking values in the space of linear but not necessarily continuous functionals on $G$, satisfying the following conditions:

$$
\begin{aligned}
& \operatorname{Im} Q_{t}^{\frac{1}{2}} \subseteq \operatorname{Dom} \varphi_{t}, \quad \varphi_{t}\left(Q_{t}^{\frac{1}{2}}\right) \in G^{*}, \quad \forall t \in[0, S], \\
& \int_{0}^{\bar{S}}\left|\varphi_{t}\left(Q_{t}^{\frac{1}{2}}\right)\right|_{G^{*}}^{2} d t<\infty .
\end{aligned}
$$

The stochastic integral of $\varphi$ with respect to $\left(\hat{P}_{t}\right)$ is denoted by $\int\left(\varphi_{s}, d \hat{P}_{s}\right)$. One can show that if $\hat{P}$ is given by (2.21) then

$$
\int_{0}^{t}\left(\varphi_{s}, d \hat{P}_{s}\right)=\int_{0}^{t}<\Gamma_{s}^{*} \circ \varphi_{s}, d W_{s}>_{l^{2}, l^{2}}, \quad t \in[0, \bar{S}]
$$

where $\Gamma_{s}^{*} \circ \varphi_{s}$ is defined by the formula $\Gamma_{s}^{*} \circ \varphi_{s}(u)=\varphi_{s}\left(\Gamma_{s} u\right), u \in l^{2}$. 
If we enlarge the class of processes, in which we search solutions of (3.28), to all stochastically integrable processes, then, under some assumptions, the structural equation (3.28) has a solution, see the theorem and the example below. Nevertheless, this solution does not have a natural interpretation as a strategy and should be treated as a mathematical idealization.

Theorem 4.1 If the operator $\Gamma$ is injective $\mathbb{P} \otimes \lambda$ a.s. then the equation (3.28) has a solution in the class of integrable processes satisfying

$$
\mathbf{E}\left(\int_{0}^{\bar{S}}\left|\varphi_{t}\left(Q_{t}^{\frac{1}{2}}\right)\right|_{G^{*}}^{2} d t\right)<\infty .
$$

Proof: Fix $\psi$ a predictable, $l^{2}$-valued process satisfying condition $\mathbf{E} \int_{0}^{\bar{S}}\left|\psi_{s}\right|_{l^{2}}^{2} d s<\infty$. We will find an integrable process $\varphi$ satisfying (4.38) such that

$$
\int_{0}^{\bar{S}}<\psi_{s}, d W_{s}>_{l^{2}, l^{2}}=\int_{0}^{\bar{S}}\left(\varphi_{s}, d \hat{P}_{s}\right)
$$

Since $\Gamma_{t}$ is injective, so

$$
\varphi_{t}(v):=<\psi_{t}, \Gamma_{t}^{-1} v>_{l^{2}}, \quad \forall v \in \operatorname{Im} \Gamma_{t}
$$

is a well defined linear functional. The process $\left(\varphi_{t}\left(\Gamma_{t}\right)\right)$ is predictable and for any $u \in l^{2}$ we have:

$$
<\psi_{t}, u>_{l^{2}}=<\psi_{t}, \Gamma_{t}^{-1} \Gamma_{t} u>_{l^{2}}=\varphi_{t}\left(\Gamma_{t} u\right) .
$$

Therefore

$$
\psi_{t}=\varphi_{t}\left(\Gamma_{t}\right), \quad \forall t \in[0, \bar{S}]
$$

so by (4.37) the formula 4.39) is satisfied.

Now, we give an example of a bond market with deterministic volatility (Gaussian HJM-model) in which the equation (3.28) has a solution.

Example 4.2 Let $\sigma^{j}, j=1,2, \ldots$ be given by the formula:

$$
\sigma^{j}(t, T):=\gamma_{j} \sin \left(j \pi\left(\frac{T-t}{\bar{S}-t}\right) \vee 0\right), \quad 0 \leq t, T \leq \bar{S}
$$

where $\gamma_{j}>0$, and $\sum_{j=1}^{\infty} \gamma_{i}^{2}<\infty$. Notice that for any $t \in[0, \bar{S}]$ the sequence $\left(\sigma^{j}(t, \cdot)\right)_{j}$ is an orthogonal system in $L^{2}[t, \bar{S}]$ and

$$
\left|\sigma^{j}(t, \cdot)\right|_{L^{2}[0, \bar{S}]} \leq \frac{\bar{S}}{2} \gamma_{j}^{2}
$$


Hence the process $\left(\sigma_{t}\right)$ satisfies (2.6). So the deterministic process $\left(\Gamma_{t}\right)$ satisfies (2.15). Therefore the corresponding process $\left(\hat{P}_{t}\right)$ is a $G$-valued martingale. To prove that in this case the equation (3.28) has a solution, it is enough, by Theorem 4.1, to show that $\Gamma_{t}$ is injective for all $t \in[0, \bar{S}]$. To this end we prove that if $\Gamma_{t} u=0$ for some $u \in l^{2}$, then $\mathrm{u}=0$. Differentiating

$$
\Gamma_{t} u(T)=-\sum_{j=1}^{\infty}\left(\int_{0}^{T} \sigma^{j}(t, s) d s\right) u^{j}
$$

with respect to $T$, we see that

$$
\sum_{j=1}^{\infty} \sigma^{j}(t, s) u^{j}=0
$$

in the sense of $L^{2}[t, \bar{S}]$. By orthogonality of the sequence $\left(\sigma^{j}(t, \cdot)\right)_{j}$ we obtain that $u^{j}=0$ for $j=1,2, \ldots$, hence $u=0$. So, $\Gamma$ is injective.

Remark 4.3 In general if $\left(\Gamma_{t}\right)$ is not injective $\mathbb{P} \otimes \lambda$ a.s., then in a similar way as in the proof of Theorem 3.2 one can show that equation (3.28) has no solution even in the class of integrable processes satisfying (4.38).

\section{References}

[1] AIHARA, S.I., A. BAGCHI (2005): "Stochastic hyperbolic dynamics for infinitedimensional forward rates and option pricing", Mathematical Finance, Vol.15, 27-47.

[2] BJÖRK, TH., G. DI MASI, Y. KABANOV and W. RUNGGALDIER (1997): "Towards a general theory of bond markets", Finance and Stochastic, 1, 141-174.

[3] BJÖRK, TH., Y. KABANOV and W. RUNGGALDIER (1997): "Bond market structure in the presence of marked point processes", Mathematical Finance, 7, 211-239.

[4] CARMONA, R., M. TEHRANCHI (2004): "A characterization of hedging portfolios for interest rate contingent claims", Annals of Applied Probability, 14, 1267-1294.

[5] DA PRATO, G., J. ZABCZYK (1992): Stochastic Equations in Infinite Dimensions, Cambridge University Press.

[6] DE DONNO, M., M. PRATELLI (2004): "On the use of measured-valued strategies in bond markets", Finance and Stochastics, 8, 87-109.

[7] DELBAEN, F., W. SCHACHERMAYER (1994): "A general version of the fundamental theorem of asset pricing", Mathematische Annalen 300, 463-520.

[8] DELBAEN, F., W. SCHACHERMAYER (1998): "The fundamental Theorem of Asset Pricing for unbounded stochastic processes", Mathematische Annalen 312, 215-250. 
[9] HEATH, D., R. JARROW AND A. MORTON (1992): "Bond pricing and the term structure of interest rates: a new methodology", Econometrica 60, 77-101.

[10] HUNT, P.J., J.E. KENNEDY (2005): "Financial Derivatives in Theory and Practice", John Wiley \& Sons, Ltd.

[11] JAKUBOWSKI, J., J. ZABCZYK (2007): "Exponential moments for HJM models with jumps", Finance and Stochastics, 11, 429-445.

[12] JARROW, R.A., D.B. MADAN (1991): "A characterization of complete security markets on a Brownian filtration", Mathematical Finance, Vol.1, 31-43.

[13] KALLENBERG, O. (2001): "Foundations of Modern Probability", 2nd ed. SpringerVerlag.

[14] KARATZAS, I., S.E. SHREVE (1998): "Methods of mathematical finance", New York Springer.

[15] LIPCER, R.S., A.N. SHIRYAEV (2001): "Statistics of random processes, I. General theory", 2nd ed. Springer-Verlag.

[16] MÉTIVIER M. (1982): "Semimartingales: a course on stochastic processes", Berlin, New York: Walter de Gruyter.

[17] MIKULEVICIUS, R., B.L. ROZOVSKII (1998): "Normalized stochastic integrals in topological vector spaces", Séminaire de probabilités XXXII (Lecture notes in Mathematics), Berlin, Heidelberg, New York Springer.

[18] MIKULEVICIUS, R., B.L. ROZOVSKII (1999): "Martingales problems for stochastic PDE's", In Carmona, R., Rozovski, B. (eds) Stochastic partial differential equations: Six perspectives. (Math. Surveys and Monographs, 64) Amer. Math. Soc., 243-326.

[19] PESZAT, SZ., J. ZABCZYK (2007): "Stochastic partial differential equations with Lévy noise", Cambridge University Press.

[20] TAFLIN, E. (2005): "Bond market completeness and attainable contingent claims", Finance and Stochastics, 9, 429 - 452. 